\title{
Magneto-optical Kerr effect studies with classical electromagnetic theory
}

Rui-Can Ma, Ling-Ling Gao, Ting Zhang, Yi Jin

Rui-Can Ma, Ling-Ling Gao, Ting Zhang, Yi Jin, "Magneto-optical Kerr effect studies with classical electromagnetic theory," Proc. SPIE 10452, 14th Conference on Education and Training in Optics and Photonics: ETOP 2017, 104525A (16 August 2017); doi: 10.1117/12.2269711

SPIE Event: 14th Conference on Education and Training in Optics and Photonics, ETOP 2017, 2017, Hangzhou, China 


\title{
MAGNETO-OPTICAL Kerr effect studies with classical electromagnetic theory
}

\author{
Rui-Can Ma ${ }^{\mathrm{a}}$, Ling-Ling Gao ${ }^{\mathrm{a}}$, Ting Zhang ${ }^{\mathrm{a}}$, Yi Jin*a \\ ${ }^{a}$ School of Physics and technology, University of Jinan/City Jinan, No.336, Nanxinzhuang west \\ road, P.R.China, 250022
}

\begin{abstract}
Employing the basic law of electromagnetic wave propagation with the constitutive relation of ideal ferromagnetic medium, the magneto-optical Kerr effect of linearly polarized plane wave on the surface of ideal ferromagnetic medium is studied. We also discuss the change law of the Kerr rotation angle with the incident angle and magnetic-field strength in detail, which can provide crucial information for the applications of the magneto-optical Kerr effect in many fields.
\end{abstract}

Keywords: magneto-optical Kerr effect, Kerr rotation angle, ideal ferromagnetic medium, linearly polarized plane wave, boundary conditions

\section{INTRODUCTION}

The magneto-optical Kerr effect ${ }^{[1]}$ refers to the phenomenon that the vibrating surface of reflected wave rotates while the incidence of the linearly polarized plane wave (LPPW) on the magnetized material, which is discovered in 1876 by $\mathrm{J} \cdot$ Kerr. Magneto-optical Kerr effect is widely used in many fields, like magnetic ordering, magnetic anisotropy, coupling between the layers of multilayer film, and the research of the magnetic ultrathin membrane phase transition behavior ${ }^{[2-5]}$. Magneto-optical Kerr effect is an important experimental methods of magnetism, and is also an effective way to measure the properties of materials, especially the physical properties of film materials ${ }^{[6-7]}$. Besides, magneto-optical information storage is the technological innovation developed in recent years, and it is a hot research field of developing practical magnetic medium material with much more superior performance for information storage. Magneto-optical Kerr effect has been applied to the high density of computer memory, e.g., there is the recordable compact disc with the storage of hundreds to thousands of megabytes in single piece.

At present, the magneto-optical Kerr effect is heavily studied in experimental measurements ${ }^{[8-11]}$. The theoretical explanation on this effect is mainly based on complex quantum theories ${ }^{[12-15]}$, while how to employ the classical electromagnetic theory on this effect is rarely discussed. In this paper, with Maxwell's electromagnetic equations, the boundary conditions ${ }^{[16]}$ and medium constitutive relation of ideal ferromagnetic medium, we analyze the LPPW reflection behavior on the surface of the ideal ferromagnetic medium, which can naturally gives the poloidal, longitudinal and transverse magneto-optic Kerr effect, besides the Kerr rotation angle change law with the incident angle and magnetic-field strength in detail. The studies can provide crucial information for the applications of the magnetooptical Kerr effect in many fields.

\section{PRIMARY ELECTROMAGNETIC THEORY AND IDEAL FERROMAGNETIC MEDIUM MODEL}

The effect of the medium on electromagnetic waves is generally described by Maxwell's differential equations:

$$
\nabla \times \vec{E}=-\frac{\partial \vec{B}}{\partial t}, \quad \nabla \times\left(\frac{\vec{B}}{\mu_{0}}-\vec{M}\right)=\vec{J}+\frac{\partial \vec{D}}{\partial t}, \quad \nabla \cdot \vec{D}=\rho, \quad \nabla \cdot \vec{B}=0 .
$$

*ss_jiny@ujn.edu.cn; phone +8613869103390

14th Conference on Education and Training in Optics and Photonics: ETOP 2017, edited by Xu Liu,

Xi-Cheng Zhang, Proc. of SPIE Vol. 10452, 104525A · C 2017 ICO, IEEE, OSA, SPIE

CCC code: $0277-786 X / 17 / \$ 18 \cdot$ doi: $10.1117 / 12.2269711$

Proc. of SPIE Vol. 10452 104525A-1 
and the corresponding boundary conditions:

$$
E_{1 t}=E_{2 t}, \quad B_{2 t}-B_{1 t}=\mu_{0}\left(M_{2 t}-M_{1 t}\right), \quad D_{2 n}=D_{1 n}, \quad B_{2 n}=B_{1 n} .
$$

Note here supposed that no free charges and free currents at the interface between two kinds of mediums. Here the ideal ferromagnetic medium referred to in this paper has the following electromagnetic properties:

$$
\vec{B}=\mu \vec{H}+\mu_{0} \vec{M}_{0},
$$

where $\vec{M}_{0}$ is a constant vector. The equation (1) is the approximation model of magnetic hysteresis loop of ferromagnetic medium. From (1) we can see that, an ideal ferromagnetic medium can be taken as a mixture of the linear magnetic medium and permanent magnet. Therefore, when electromagnetic behaviors are relevant to the ideal ferromagnetic medium, the corresponding analysis can be divided into two steps: at first, the studies are made according to the linear magnetic medium of $\vec{B}=\mu \vec{H}$, and then the influence and modification of the permanent magnet is considered carefully. In the next section, employing the above ideas, we shall deduce the magneto-optical Kerr effects and rotation angles when LPPW is incident to the surface of ideal ferromagnetic medium, in which the $\vec{M}_{0}$ can be along one of the poloidal, longitudinal and transverse directions.

\section{MAGNETO-OPTICAL KERR EFFECTS ON IDEAL FERROMAGNETIC MEDIUM SURFACE}

Figure1 shows the coordinate system employed in the following derivations. The XOY plane is the interface of two semi-infinite mediums, where 2 is the ideal ferromagnetic medium and 1 is the homogeneous linear medium. The normal direction of the interface is consistent with the $\mathrm{Z}$ axis. The incidence of LPPW is from 1 to 2, so the incident plane is $\mathrm{XOZ}$ plane. Here the Kerr rotation angle $\beta$ is defined as the included angle between the vector direction of total magnetic field in reflected wave and the incident plane.

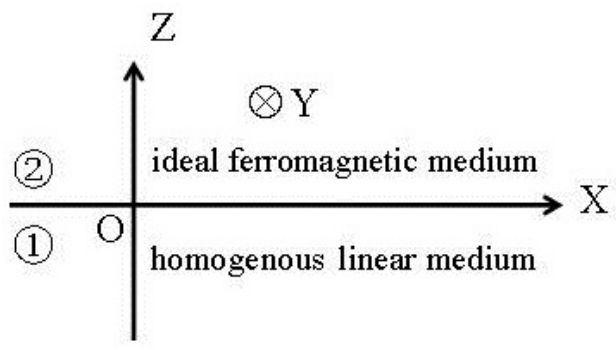

Figure 1. The coordinate system is adopted.

\subsection{S-wave case}

In S-wave case, the electric/magnetic field vibration direction of incident wave is perpendicular/parallel to the incident plane. To obtain the magnetic field amplitude ratios of reflected and refracted waves to incident wave, one needs two boundary conditions: tangential components of electric fields are continuous, and tangential components of magnetic fields are continuous on the interface, too. As mentioned in Section 2, these boundary conditions correspond respectively to the following formulas (2)-(3):

$$
\begin{gathered}
E+E^{\prime}=E^{\prime \prime}, \\
H \cos \theta-H^{\prime} \cos \theta=H^{\prime \prime} \cos \theta^{\prime \prime},
\end{gathered}
$$

where $E / H$ is the electric/magnetic field amplitude of the incident wave, and $E^{\prime} / H^{\prime}\left(E^{\prime \prime} / H^{\prime \prime}\right)$ is the electric/magnetic field amplitude of reflected (refracted) wave when supposed 2 is linear medium, so according to TEM wave properties we have 


$$
E=\sqrt{\frac{\mu_{1}}{\varepsilon_{1}}} H, \quad E^{\prime}=\sqrt{\frac{\mu_{1}}{\varepsilon_{1}}} H^{\prime}, \quad E^{\prime \prime}=\sqrt{\frac{\mu_{2}}{\varepsilon_{2}}} H^{\prime \prime} .
$$

Here the tangential direction is $\mathrm{X}$ or $\mathrm{Y}$ axis as shown in Figure 1. With Eq. (2)-(4), we can obtain the equation on $H^{\prime}$ of reflected wave:

$$
H \cos \theta-H^{\prime} \cos \theta^{\prime}=\sqrt{\frac{\varepsilon_{2} \mu_{1}}{\varepsilon_{1} \mu_{2}}}\left(H+H^{\prime}\right) \cos \theta^{\prime \prime} .
$$

According to refraction law: $\frac{\sin \theta}{\sin \theta^{\prime \prime}}=\sqrt{\frac{\mu_{2} \varepsilon_{2}}{\mu_{1} \varepsilon_{1}}}$, we can get

$$
\cos \theta^{\prime \prime}=\sqrt{1-\frac{\mu_{1} \varepsilon_{1}}{\mu_{2} \varepsilon_{2}} \sin ^{2} \theta}
$$

With Eq. (5) and (6), $H^{\prime}$ is solved as

$$
H^{\prime}=\frac{\sqrt{\frac{\mu_{2}}{\varepsilon_{2}}} \cos \theta-\sqrt{\frac{\mu_{1}}{\varepsilon_{1}}} \sqrt{1-\frac{\mu_{1} \varepsilon_{1}}{\mu_{2} \varepsilon_{2}} \sin ^{2} \theta}}{\sqrt{\frac{\mu_{2}}{\varepsilon_{2}}} \cos \theta+\sqrt{\frac{\mu_{1}}{\varepsilon_{1}}} \sqrt{1-\frac{\mu_{1} \varepsilon_{1}}{\mu_{2} \varepsilon_{2}} \sin ^{2} \theta}} H .
$$

In the above process of derivation, only the linear part $\mu \vec{H}$ of electromagnetic characteristic law of ideal ferromagnetic medium in Eq. (1), i.e., the medium-2 is supposed just as linear medium. However, the fundamental reason of ideal ferromagnetic medium different from linear medium is that the term of $\mu_{0} \vec{M}_{0}$ exists in its electromagnetic characteristics, which can have an important impact on the boundary conditions and then request the reflected wave to produce extra magnetic field besides linear medium. Furthermore, this extra magnetic field may vary with the direction of $\vec{M}_{0}$. So to get the total magnetic field $\vec{H}_{\text {tot }}^{\prime}$ of reflected wave, the specific impact of the term of $\mu_{0} \vec{M}_{0}$ should be considered and the magnetic field boundary conditions should be modified correspondingly.

When $\vec{M}_{0}$ is in X direction (i.e., lengthwise direction), the boundary condition that the tangential components of magnetic field are continuous can be written as:

$$
H \cos \theta-H_{t o t}^{\prime} \cos \theta=H^{\prime \prime} \cos \theta^{\prime \prime}+M_{0},
$$

With Eq. (2), (3'), (4) and (6), we get

$$
H_{t o t}^{\prime}=\frac{H \cos \theta-H \sqrt{\frac{\mu_{1} \varepsilon_{2}}{\mu_{2} \varepsilon_{1}}} \sqrt{1-\frac{\mu_{1} \varepsilon_{1}}{\mu_{2} \varepsilon_{2}} \sin ^{2} \theta}-M_{0}}{\cos \theta+\sqrt{\frac{\mu_{1} \varepsilon_{2}}{\mu_{2} \varepsilon_{1}}} \sqrt{1-\frac{\mu_{1} \varepsilon_{1}}{\mu_{2} \varepsilon_{2}} \sin ^{2} \theta}} .
$$

In this case, the direction of $\vec{H}_{t o t}^{\prime}$ is parallel to the incident plane (XOZ plane), so the Kerr rotation angle is zero.

When $\vec{M}_{0}$ is in the Y axis (i.e., transverse direction), the reflected wave's total magnetic field is

$$
\vec{H}_{t o t}^{\prime}=-H^{\prime} \cos \theta \cdot \vec{i}+M_{0} \cdot \vec{j}-H^{\prime} \sin \theta \cdot \vec{k}
$$


In this case, the direction of $\vec{H}_{t o t}^{\prime}$ is not parallel to the incident plane, and the Kerr rotation angle is

$$
\beta=\arctan \left(\frac{\left(\sqrt{\frac{\mu_{2}}{\varepsilon_{2}}} \cos \theta+\sqrt{\frac{\mu_{1}}{\varepsilon_{1}}} \sqrt{1-\frac{\mu_{1} \varepsilon_{1}}{\mu_{2} \varepsilon_{2}} \sin ^{2} \theta}\right) M_{0}}{\left(\sqrt{\frac{\mu_{2}}{\varepsilon_{2}}} \cos \theta-\sqrt{\frac{\mu_{1}}{\varepsilon_{1}}} \sqrt{1-\frac{\mu_{1} \varepsilon_{1}}{\mu_{2} \varepsilon_{2}} \sin ^{2} \theta}\right) H} .\right.
$$

When $\vec{M}_{0}$ is along $\mathrm{Z}$ direction (i.e., poloidal direction), one needs not only the boundary conditions of (2) and (3), but also the boundary condition that the normal components of magnetic field are continuous, that is

$$
B \sin \theta+B_{t o t}^{\prime} \sin \theta=B_{t o t}^{\prime \prime} \sin \theta \text {. }
$$

Then we can obtain

$$
\vec{H}_{t o t}^{\prime}=-H^{\prime} \cos \theta \cdot \vec{i}+\left(-H^{\prime} \sin \theta+\frac{\mu_{0} M_{0}}{\mu_{1}}\right) \vec{k} .
$$

Here, like $\vec{M}_{0}$ is in the $\mathrm{X}$ direction, $\vec{H}_{t o t}^{\prime}$ is parallel to the incident plane and the Kerr rotation angle is zero.

\subsection{P-wave case}

In this case, the electric/magnetic field vibration direction of incident wave is parallel/perpendicular to the incident plane. When we just take account of the linear part of electromagnetic characteristic law of ideal ferromagnetic medium, with the boundary conditions that tangential components of electric fields and magnetic fields are continuous separately on the interface, $H^{\prime}$ is solved as

$$
H^{\prime}=\frac{\sqrt{\frac{\mu_{1}}{\varepsilon_{1}}} \cos \theta-\sqrt{\frac{\mu_{2}}{\varepsilon_{2}}} \sqrt{1-\frac{\mu_{1} \varepsilon_{1}}{\mu_{2} \varepsilon_{2}} \sin ^{2} \theta}}{\sqrt{\frac{\mu_{1}}{\varepsilon_{1}}} \cos \theta+\sqrt{\frac{\mu_{2}}{\varepsilon_{2}}} \sqrt{1-\frac{\mu_{1} \varepsilon_{1}}{\mu_{2} \varepsilon_{2}} \sin ^{2} \theta}} H .
$$

The derivation with no more detailed description is similar as in the S-wave case.

When $\vec{M}_{0}$ is along the $\mathrm{X}$ axis, we can obtain the total magnetic field of the reflected wave as

$$
\vec{H}_{t o t}^{\prime}=M_{0} \cdot \vec{i}+H^{\prime} \cdot \vec{j},
$$

and the Kerr rotation angle is

$$
\beta=\arctan \left(\frac{\left(\sqrt{\frac{\mu_{1}}{\varepsilon_{1}}} \cos \theta-\sqrt{\frac{\mu_{2}}{\varepsilon_{2}}} \sqrt{1-\frac{\mu_{1} \varepsilon_{1}}{\mu_{2} \varepsilon_{2}} \sin ^{2} \theta}\right) H}{\left(\sqrt{\frac{\mu_{1}}{\varepsilon_{1}}} \cos \theta+\sqrt{\frac{\mu_{2}}{\varepsilon_{2}}} \sqrt{1-\frac{\mu_{1} \varepsilon_{1}}{\mu_{2} \varepsilon_{2}} \sin ^{2} \theta}\right) M_{0}}\right) .
$$

When $\vec{M}_{0}$ is in the $\mathrm{Y}$ axis, the total magnetic field of the reflected wave is

$$
\vec{H}_{t o t}^{\prime}=\left(H^{\prime}+M_{0}\right) \vec{j}
$$

so the vector of $\vec{H}_{t o t}^{\prime}$ is still within the incident plane and the Kerr rotation angle is zero. 
When $\vec{M}_{0}$ is in the $\mathrm{Z}$ axis, after taking account of the boundary condition that the normal components of magnetic field are continuous besides, one gets reflected wave's total magnetic field as

$$
\vec{H}_{t o t}^{\prime}=H^{\prime} \cdot \vec{j}+\frac{\mu_{0} M_{0}}{\mu_{1}} \cdot \vec{k}
$$

then the Kerr rotation angle is

$$
\beta=\arctan \left(\frac{\mu_{1}\left(\sqrt{\frac{\mu_{1}}{\varepsilon_{1}}} \cos \theta-\sqrt{\frac{\mu_{2}}{\varepsilon_{2}}} \sqrt{\left.1-\frac{\mu_{1} \varepsilon_{1}}{\mu_{2} \varepsilon_{2}} \sin ^{2} \theta\right) H}\right.}{\mu_{0}\left(\sqrt{\frac{\mu_{1}}{\varepsilon_{1}}} \cos \theta+\sqrt{\frac{\mu_{2}}{\varepsilon_{2}}} \sqrt{1-\frac{\mu_{1} \varepsilon_{1}}{\mu_{2} \varepsilon_{2}} \sin ^{2} \theta}\right) M_{0}}\right) .
$$

\section{RESULTS AND DISCUSSION}

Based on the analysis in Section 3, we can draw a conclusion that magneto-optical Kerr effect is generated when LPPW propagates on the surface of ideal ferromagnetic medium. The Kerr rotation angle $\beta$ value is related with the incident angle $\vec{M}_{0}$ and magnetic field $H$, and with the different directions of $\vec{M}_{0}$ the corresponding change laws are different, as shown in Table 1.

\begin{tabular}{|c|c|c|}
\hline $\begin{array}{c}\vec{M}_{0} \\
\text { direction }\end{array}$ & S-wave & P-wave \\
\hline$\frac{\mathrm{X}}{\text { (longitudinal) }}$ & $\beta=0$, no rotation & $\beta=\arctan \frac{\left(\sqrt{\frac{\mu_{1}}{\varepsilon_{1}}} \cos \theta-\sqrt{\frac{\mu_{2}}{\varepsilon_{2}}} \sqrt{1-\frac{\mu_{1} \varepsilon_{1}}{\mu_{2} \varepsilon_{2}} \sin ^{2} \theta}\right) H}{\left(\sqrt{\frac{\mu_{1}}{\varepsilon_{1}}} \cos \theta+\sqrt{\frac{\mu_{2}}{\varepsilon_{2}}} \sqrt{1-\frac{\mu_{1} \varepsilon_{1}}{\mu_{2} \varepsilon_{2}} \sin ^{2} \theta}\right) M_{0}}$ \\
\hline $\begin{array}{c}\mathrm{Y} \\
\text { (transverse) }\end{array}$ & $\beta=\arctan \frac{\left(\sqrt{\frac{\mu_{2}}{\varepsilon_{2}}} \cos \theta+\sqrt{\frac{\mu_{1}}{\varepsilon_{1}}} \sqrt{1-\frac{\mu_{1} \varepsilon_{1}}{\mu_{2} \varepsilon_{2}} \sin ^{2} \theta}\right) M_{0}}{\left(\sqrt{\frac{\mu_{2}}{\varepsilon_{2}}} \cos \theta-\sqrt{\frac{\mu_{1}}{\varepsilon_{1}}} \sqrt{1-\frac{\mu_{1} \varepsilon_{1}}{\mu_{2} \varepsilon_{2}} \sin ^{2} \theta}\right) H}$ & $\beta=0$, no rotation \\
\hline Z (poloidal) & $\beta=0$, no rotation & $\beta=\arctan \frac{\left(\sqrt{\frac{\mu_{1}}{\varepsilon_{1}}} \cos \theta-\sqrt{\frac{\mu_{2}}{\varepsilon_{2}}} \sqrt{1-\frac{\mu_{1} \varepsilon_{1}}{\mu_{2} \varepsilon_{2}} \sin ^{2} \theta}\right) H}{\left(\sqrt{\frac{\mu_{1}}{\varepsilon_{1}}} \cos \theta+\sqrt{\frac{\mu_{2}}{\varepsilon_{2}}} \sqrt{1-\frac{\mu_{1} \varepsilon_{1}}{\mu_{2} \varepsilon_{2}} \sin ^{2} \theta}\right) M_{0}}$ \\
\hline
\end{tabular}

Table 1. The Kerr rotation angle $\beta$ with $\vec{M}_{0}$ along different directions.

According to Table 1 , we can see that for S-wave the Kerr rotation angle $\beta \neq 0$, and on the contrary $\beta=0$ for P-wave only when $\vec{M}_{0}$ along the $\mathrm{Y}$ axis (transverse direction). Furthermore, when $\vec{M}_{0}$ is along the $\mathrm{X}$ or $\mathrm{Z}$ axis, the expressions of $\beta$ are same for P-wave. 
For simplicity, the following analysis on the variation trends of the Kerr rotation angle with the incident angle $\theta$ and magnetic field $H$, is in the conditions of the LPPW incident on the ideal ferromagnetic medium from vacuum, namely medium-1 is vacuum. Therefore, the magnetic conductivity and dielectric constant of medium- 1 and medium- 2 are respectively adopted as: $\mu_{1}=\mu_{0}=4 \pi \times 10^{-7} \mathrm{H} / \mathrm{m}, \varepsilon_{1}=\varepsilon_{0}=8.85 \times 10^{-12} \mathrm{~F} / \mathrm{m}$, and $\mu_{2}=500 \times 4 \pi \times 10^{-7} \mathrm{H} / \mathrm{m}, \varepsilon_{2}=5.51 \times 8.85 \times 10^{-12} \mathrm{~F} / \mathrm{m}$. Here $\mu_{2}$ and $\varepsilon_{2}$ of ideal ferromagnetic medium are taken the values as manganese zinc ferrite, and according to its hysteresis loop of manganese, $M_{0}$ is estimated as $M_{0}=4 \times 10^{4} \mathrm{~A} / \mathrm{m}$. After substituting above constant values into Table 1 , one can get the functions of $\beta$ only related with two variables: the incident angle $\theta$ and magnetic field $H$, as shown in Figure 2 .
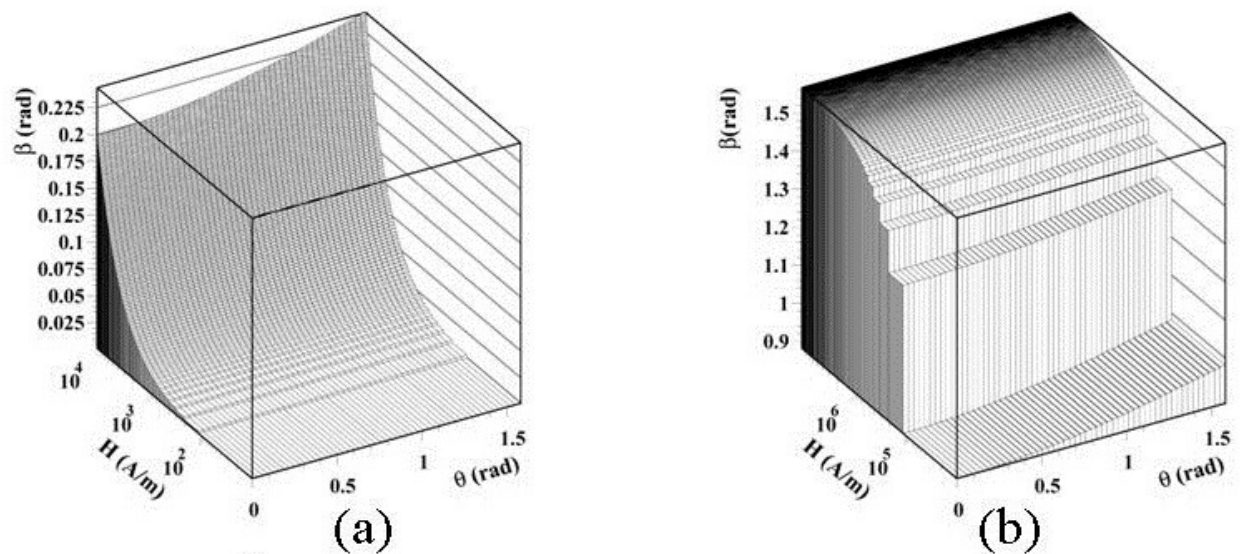

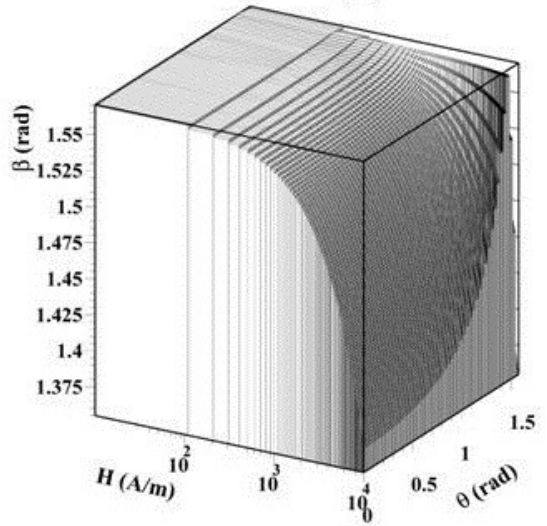

(c)

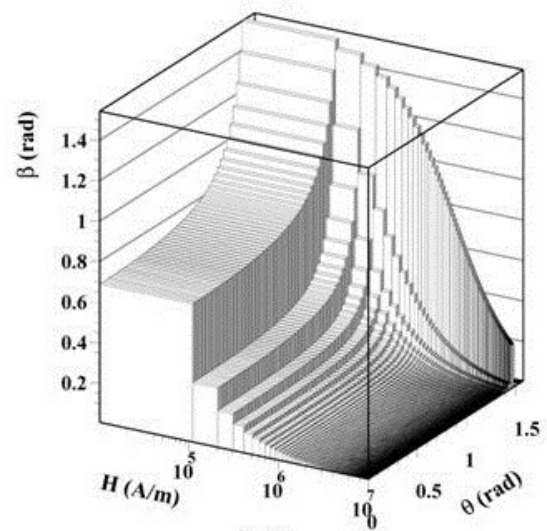

(d)

Figure 2. The 3D Figure of the function of the Kerr rotation angle $\beta$ with two variables of incident angle $\theta(0 \sim \pi / 2)$ and magnetic field $H$ : (a) $\vec{M}_{0}$ direction is along the $\mathrm{X}(\mathrm{Z})$ axis, $H$ belongs to $10 \sim 10^{4} \mathrm{~A} / \mathrm{m}$; (b) $\vec{M}_{0}$ direction is along the $\mathrm{X}(\mathrm{Z})$ axis, $H$ belongs to $10^{4} \sim 10^{7} \mathrm{~A} / \mathrm{m}$; (c) $\vec{M}_{0}$ direction is along the $\mathrm{Y}$ axis, $H$ belongs to $10 \sim 10^{4} \mathrm{~A} / \mathrm{m}$; (d) $\vec{M}_{0}$ direction is along the $\mathrm{Y}$ axis, $H$ belongs to $10^{4} \sim 10^{7} \mathrm{~A} / \mathrm{m}$. Here we ignore other interaction effects of wave with substances. 


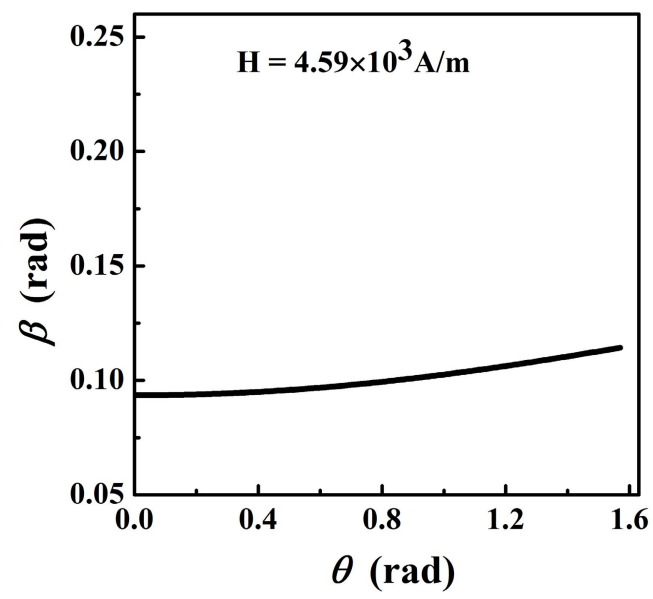

(a)

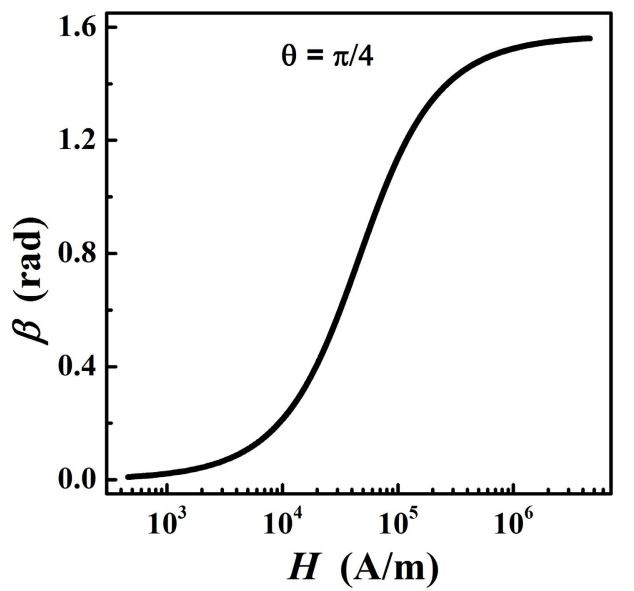

(b)

Figure 3. When $\vec{M}_{0}$ direction is along the $\mathrm{X}(\mathrm{Z})$ axis, the variation trend of the Kerr rotation angle $\beta$ with (a) the incident angle $\theta$, (b) the magnetic field $H$ of incident P-wave.

In the following we shall give the detailed analysis of $\beta$ dependence of $H(\theta)$ when regarding $\theta(H)$ as a constant, see Figure 3 and 4 respectively.

If the magnetic field $H$ of incident wave is adopted as $H=4.59 \times 10^{3} \mathrm{~A} / \mathrm{m}$ (here we ignore other interaction effects of wave with substances) ${ }^{[17]}$, the refraction law $\frac{\sin \theta}{\sin \theta^{\prime \prime}}=\sqrt{\frac{\mu_{2} \varepsilon_{2}}{\mu_{0} \varepsilon_{0}}}$ and $\sin ^{2} \theta+\cos ^{2} \theta=1$ are also considered, for $\vec{M}_{0}$ direction along the $\mathrm{X}(\mathrm{Z})$ axis $\beta$ can be simplified as

$$
\beta \approx \arctan \left(1.15 \times 10^{-2} \times\left|1-\frac{20}{\cos \theta+10}\right|\right),
$$

according to Table 1. $\beta \in[0.09,0.11]$ because of $\theta \in[0, \pi / 2]$. The $\beta$ is defined as the included angle between the vector direction of total magnetic field in reflected wave and the incident plane, it should not be greater than $\pi / 2 \mathrm{rad}$, therefore the absolute value is adopted in (9). Figure 3(a) shows that $\beta$ is monotone increasing very slowly with $\theta$ increasing, which can be easily understood when considering Eq. (9) and tangent function variation curve with $\cos \theta \in[0,1]$. On the other hand, when the incident angle $\theta$ is considered as a constant $\pi / 4 \mathrm{rad}$, Figure 3(b) indicates $\beta$ changes with $H$ as Eq.(10)

$$
\beta=\arctan \left(\frac{1.41-20 H}{1.41+8 \times 10^{5}}\right) .
$$

It increases very slowly when $H$ belongs to $0 \sim 10^{3} \mathrm{~A} / \mathrm{m}$, but rapidly increases when $H$ belongs to $10^{3} \sim 10^{6} \mathrm{~A} / \mathrm{m}$, and it trends to a stable value of $\pi / 2$ rad when $H \geq 10^{6} \mathrm{~A} / \mathrm{m}$. 


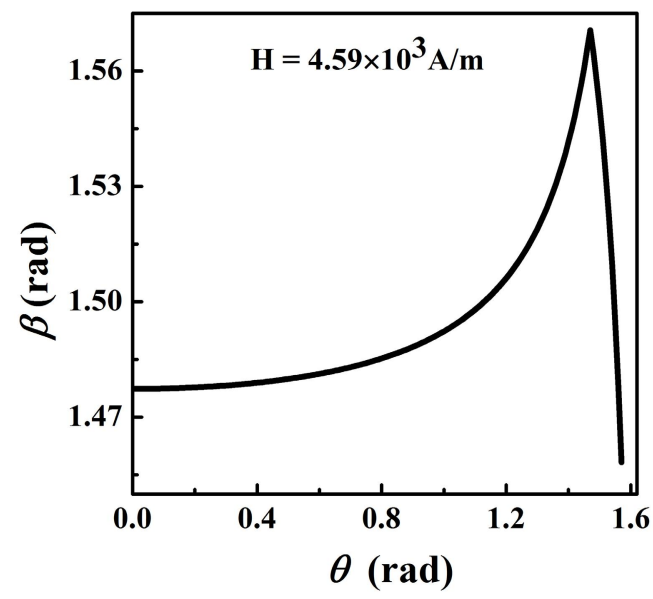

(a)

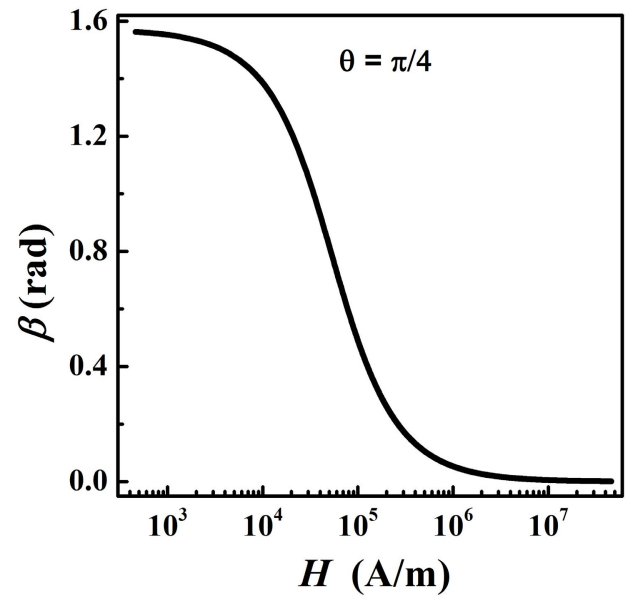

(b)

Figure 4. When $\vec{M}_{0}$ direction is along the Y axis, the variation trend of the Kerr rotation angle $\beta$ with (a) the incident angle $\theta$, (b) the magnetic field $H$ of incident S-wave.

For $\vec{M}_{0}$ direction along the $\mathrm{Y}$ axis and $H=4.59 \times 10^{3} \mathrm{~A} / \mathrm{m}$, according to Table 1 , the Kerr rotation angle $\beta$ can be simplified as

$$
\beta \approx \arctan \left(4.19 \times 10^{-2} \times\left|1+\frac{2}{10 \cos \theta-1}\right|\right)
$$

When the incident $\theta \in[0, \pi / 2], \beta \in[1.46, \pi / 2]$ within a very small range of about 0.1 rad. In Eq. (11), because there is a term of $(10 \cos \theta-1)$ on the denominator, we can see that with the increase of $\theta$, the value of this term changes from positive to 0 and then to negative, i.e., the direction of the total magnetic field of reflected wave will have a reversal. However, according to the definition of $\beta$, here we also take the absolute value in Eq. (11). Figure 4(a) shows that when $\theta$ increases from 0 , according to cosine function properties, $\beta$ is about $1.48 \mathrm{rad}$ and the increase is not obviously at the beginning. When $\theta$ near $1.47 \mathrm{rad}, \beta$ rapidly increases to $\pi / 2 \mathrm{rad}$, and then sharp decreases to about $1.46 \mathrm{rad}$ after $\theta$ continues to increase. The location of peak value of $\beta=\pi / 2 \mathrm{rad}$ is $\theta=1.47 \mathrm{rad}$, which is a fixed value due to the peak position is decided by the term of $\left(\sqrt{\frac{\mu_{2}}{\varepsilon_{2}}} \cos \theta-\sqrt{\frac{\mu_{1}}{\varepsilon_{1}}} \sqrt{1-\frac{\mu_{1} \varepsilon_{1}}{\mu_{2} \varepsilon_{2}} \sin ^{2} \theta}\right)$ equal to zero on the denominator of the expression of $\beta$ list in Table 1 . When the incident angle $\theta$ is considered as a constant $\pi / 4$ rad, from Figure 4(b) based on Eq.(12)

$$
\beta=\arctan \left(\frac{5.32 \times 10^{4}}{H}\right),
$$

we can see a curve with reverse trend with $H$ comparing to Figure 3(b).

\section{CONCLUSION AND OUTLOOK}

Based on the classical electromagnetic theory, we study the propagation behavior of LPPW incident on the surface of the ideal ferromagnetic medium of constitutive relation $\vec{B}=\mu \vec{H}+\mu_{0} \vec{M}_{0}$, which is different from the general linear homogeneous medium and leads to the magneto-optical Kerr effect. We discuss in detail the change law of the Kerr 
rotation angle with the incident angle and the magnetic field for $\vec{M}_{0}$ along different directions in S-wave and P-wave. The theoretical analysis indicates that the Kerr rotation angle $\beta$ has the follow characteristics: (1) for S-wave the Kerr rotation angle $\beta \neq 0$, but $\beta=0$ for $\mathrm{P}$-wave only when $\vec{M}_{0}$ along the $\mathrm{Y}$ axis (transverse direction); when $\vec{M}_{0}$ is along the $\mathrm{X}$ or $\mathrm{Z}$ axis (longitudinal or poloidal direction), the expressions of $\beta$ are same for P-wave. (2) When the incident angle $\theta$ increases and magnetic field $H$ is invariant, $\beta$ has a slow growth with $\vec{M}_{0}$ in the $\mathrm{X}$ or $\mathrm{Z}$ direction; when $\vec{M}_{0}$ direction is the $\mathrm{Y}$ axis, there is a peak value of $\beta=\pi / 2 \mathrm{rad}$ and the position of this peak does not change. (3) With the incident magnetic field $H$ increases and incident angle $\theta$ is invariant, $\beta$ increases from about 0 rad to the maximum value of $\pi / 2$ $\operatorname{rad}$ when $\vec{M}_{0}$ direction is in the $\mathrm{X}$ or $\mathrm{Z}$ axis; however, when $\vec{M}_{0}$ is along the $\mathrm{Y}$ axis $\beta$ decreases from $\pi / 2 \mathrm{rad}$ to $0 \mathrm{rad}$.

The analysis employing the classical electromagnetic theory on the magneto-optical Kerr effect in this paper can share some similarity with other investigations, e.g., the electro-optic Kerr effect, Faraday effect, and the concerned phenomena of total reflection and Brewster angle. These theoretical studies can shed light on the application of new technology related with the subjects mentioned above.

\section{REFERENCES}

[1] Minden, H. T., "Ellipsometric measurement of the Kerr magneto-optic effect," Applied Optics 8(6), 813-817 (1979).

[2] Shao-Peng Hao, Yu-Ling Song, "The Kerr effect of a dielectric/anti ferromagnetic/metal sandwiched structure," Natural Sciences Journal of Harbin Normal University 32(1), 80-83 (2016).

[3] Guo-Qian Zhang, Yon-Ming Yang and Zhen-Bin Zhang, "Using Self-made Observation Systematic of Magnetic Domain Magneto-optical Kerr" Materials Review 24(10), 115-117 (2010).

[4] Van Engen, P. G. , et al, "a material with very high magneto-optical Kerr effect," Applied Physics Letters. 42(2), 202-204 (1983).

[5] Tse, Wang-Kong, A.H. MacDonald, "Giant magneto-optical Kerr effect and universal Faraday effect in thinfilm topological insulator," Physical Review Letter 306(5703), 057401 (2004).

[6] Ying Jiang, Hong-Yu Zou, "Measuring surface magnetism by surface magneto optic Kerr effect," Journal of Liaoning Normal University 29(4), 433-434 (2006).

[7] Ping-An Liu, Fei Ding, "Magnetic hysteresis loop of the ferromagnetic film measured by using the test system of surface magneto optic Kerr effect," Jour nal of Liao ning Normal University(Natural Science Edition) 9(26),3-5 (2006).

[8] Ting-jun Ma, Ling An, and Wei-Jie Chen, "Magneto-optical Kerr effect and its measurement," Journal Harbin University of Commerce 21(6), 787-788 (2005).

[9] Feng-Xi Jin, Long Sun, and Cheng-Gui Wang, "Measurement of dispersive power of optical material with Faraday effect principle," Journal of Yanbian University 28(3), 165-167 (2002).

[10] Dong-Liang Qian, Liang-Yao Chen, et al, "A method to measure completely the magneto-optical Kerr and Faraday effects," Acta Optica Sinica 19(4), 474-480 (1999).

[11] Xin-Wei Chen, Fu-Sheng Qiu, et al, "A method for measuring completely the Faraday effects and losses in optical mirror coated," Acta Photonica Sinica 38(11), 2937-2941 (2009).

[12] Xue-Long Zhang, Guo-Ying Zhang, et al, "The classical and quantum theory of magneto-optical Faraday effect.," Guangxi Sciences 12(1), 22-24 (2005).

[13] Jia-Fu Wang, Zuo-Yi Li, et al, "A quantum scattering method for the magneto-optical Kerr effect," Journal of Huazhong University Ofence \& Technology 23(8), 19-22 (1995).

[14] Qing-Chun Zhou, Rong-Qing Xu, and Jia-Fu Qang, "Temperature dependence of magneto-optical Kerr effect," Acta Sinica Quantum Optica 7(4), 172-175 (2001).

[15] Liang-Yao Chen, "Fundamentals and measurements of magnet-optic Faraday and Kerr effects," Semiconductor Optoelectronics 12(4), 392-397 (1991).

[16] Jackson, JohnDavid, [Classical electrodynamics], Мир., 13-19 (1900).

[17] Shao-Xian Meng, "Ultrastrong laser fields physics," Progress in Physics 19(3), 236-269 (1999). 